\title{
Differential patterns of change in brain connectivity resulting from traumatic brain injury
}

Johan Nakuci ${ }^{1}$, Matthew McGuire ${ }^{1,2}$, Ferdinand Schweser ${ }^{3,4}$, David Poulsen ${ }^{2}$, Sarah F. Muldoon ${ }^{1,5}$

${ }^{1}$ Neuroscience Program, University at Buffalo, Buffalo, NY

${ }^{2}$ Department of Neurosurgery, University at Buffalo, Buffalo, NY

${ }^{3}$ Buffalo Neuroimaging Analysis Center, Department of Neurology, School of Medicine and Biomedical Sciences, University at Buffalo, Buffalo, NY

${ }^{4}$ Center for Biomedical Imaging, Clinical and Translational Science Institute, University at Buffalo, Buffalo, NY

${ }^{5}$ Department of Mathematics and CDSE Program, University at Buffalo, Buffalo, NY

\section{Abstract}

Background: Traumatic brain injury (TBI) damages white matter tracts, disrupting brain network structure and communication. There exists a wide heterogeneity in the pattern of structural damage associated with injury, as well as a large heterogeneity in behavioral outcomes. However, little is known about the relationship between changes in network connectivity and clinical outcomes.

Methods: We utilize the rat lateral fluid-percussion injury (FPI) model of severe TBI to study differences in brain connectivity in 8 animals that received the insult and 11 animals that received only a craniectomy. Diffusion Tensor Imaging (DTI) is performed 5 weeks after the injury and network theory is used to investigate changes in white matter connectivity.

Results: We find that 1) global network measures are not able to distinguish between healthy and injured animals; 2) injury induced alterations predominantly exist in a subset of connections (subnetworks) distributed throughout the brain; and 3) injured animals can be divided into subgroups based on changes in network motifs - measures of local structural connectivity. Additionally, alterations in predicted functional connectivity indicate that the subgroups have different propensities to synchronize brain activity, which could relate to the heterogeneity of clinical outcomes such as the risk of developing post-traumatic epilepsy. 
Discussion: These results suggest that network measures can be used to quantify progressive changes in brain connectivity due to injury and differentiate among subpopulations with similar injuries but different pathological trajectories.

\section{Impact Statement}

White matter tracts are important for efficient communication between brain regions and their connectivity pattern underlies proper brain function. Traumatic brain injury (TBI) damages white matter tracts and changes brain connectivity, but how specific changes relate to differences in clinical/behavioral outcomes is not known. Using network theory to study injury related changes in structural connectivity, we find that local measures of network structure can identify subgroups of injured rats with different types of changes in brain structure. Our results suggest that these different patterns of change could relate to differences in clinical outcomes such as the propensity to develop epilepsy. 


\section{Introduction}

White matter tracts provide the underlying structure for communication between brain regions and therefore damage to these tracts can have significant impact on brain function. Traumatic Brain Injury ( $\mathrm{TBI}$ ) can cause diffuse axonal injury beyond the site of the trauma, with white matter tracts particularly vulnerable to injury (Johnson et al., 2013b). The location and extent of an injury can be assessed with Diffusion Tensor Imaging (DTI) and alterations in diffusion properties can characterize structural changes in the brain (Basser et al., 1994).

In humans (Benson et al., 2007; Kraus et al., 2007; Mac Donald et al., 2007; Wilde et al., 2008; Kinnunen et al., 2011) and rodents (Mac Donald et al., 2007; Budde et al., 2011; van de Looij et al., 2012; Pischiutta et al., 2018), the diffusion metrics of fractional anisotropy (FA), axial diffusivity (AD), radial diffusivity (RD), and mean diffusivity (MD) have all shown injury related changes in brain structure and additional changes have been found in white matter fiber density (Harris et al., 2016a; Wright et al., 2017). In animal models, the changes in diffusion metrics and fiber density indicate the possibility that, alongside axonal and myelin degeneration, there is axonal sprouting and structural reorganization following an injury (Christman et al., 1997; Harris et al., 2016a). Despite the extensive research in TBI induced structural changes, thus far it has been difficult to link observed changes in brain structure to behavioral/clinical outcomes because of the extent of damage and change in brain structure is highly heterogeneous in humans and rodents.

Diffusion metrics can provide information about injury related changes to brain structure, and the use of tools from network theory (Bassett and Sporns, 2017) offers an opportunity to understand how TBI induces local and global changes in brain connectivity (Feldt et al., 2011; Sharp et al., 2014; Bassett and Sporns, 2017). Studies utilizing structural networks have found that TBI induces changes in a subset of brain connections (Hayes et al., 2016; Iraji et al., 2016; Thengone et al., 2016; Dall'Acqua et al., 2017) and can alter a network's characteristic path 
length, global/local efficiency, betweeness centrality, eigenvector centrality, and smallworldness (Caeyenberghs et al., 2014; Yuan et al., 2015; Königs et al., 2017; van der Horn et al., 2017). Furthermore, it has been shown that betweenness- and eigenvector-centrality can potentially be used as diagnostic biomarkers (Fagerholm et al., 2015).

One of the clinical outcomes of TBI is an increased in risk developing post-traumatic epilepsy (PTE) (Annegers et al., 1998). It is estimated that TBI causes $20 \%$ of symptomatic epilepsies, and $5 \%$ of all epilepsies (Englander et al., 2003). The incidence of PTE can be as high as $53 \%$ of patients depending on injury severity and type of injury (penetrating vs blunt) (Frey, 2003). Unfortunately, there are no clear biomarkers for predicting who will develop PTE (Pitkänen and Immonen, 2014).

Here, we use the lateral fluid-percussion injury (FPI) model to induce severe TBI in rats and use network theory to study the associated changes in brain structure post-injury (Mclntosh et al., 1989; Rau et al., 2012, 2014; Smith et al., 2018). This model mimics the damage observed clinically (Xiong et al., 2013) and displays a heterogeneity in the severity of injury both structurally and functionally, as some rats will go on to display spontaneous seizures(Bolkvadze and Pitkänen, 2012; Immonen et al., 2013). To characterize the heterogeneity in structural damage post-injury, we examine the properties of large-scale structural brain networks derived from dMRI imaging data in injured and control rats. We find differences in subnetwork brain structure between injured and control rats and that animals within the TBI, but not control, group can be clustered into two distinct subpopulations driven by changes in local motif structure of the brain networks. Further, simulated functional connectivity patterns predict that one subgroup shows higher levels of synchronization, which we speculate could be related to its propensity to later develop epilepsy. 


\section{Materials and Methods}

\section{Ethical Treatment of Animals}

The Institutional Animal Care and Use Committee at SUNY Buffalo approved all procedures in these studies.

\section{TBI Procedure}

Adult, male, Wistar rats (300-350g) were obtained from Charles River Laboratories (Wilmington, MA) and single housed in a 12-hour light/dark schedule with ad libitum access to food and water. The lateral fluid percussion injury procedure was performed as we have previously published (McGuire et al., 2019).

Briefly, animals were deeply anesthetized using $2-4 \%$ isoflurane. A $5 \mathrm{~mm}$ trephin was used to make a craniectomy over the right hemisphere equidistant between the lambda and the bregma and adjacent to the lateral ridge. Animals received a $20 \mathrm{~ms}$ fluid pressure pulse to the dura at an average of 2.79ATMs of atmospheres (range of 2.64 to 2.98 ATMs). Following injury, animals became apneic after injury for an average of 38 seconds (range of 0 to 60 seconds) and were manually ventilated with supplemented $\mathrm{O}_{2}$ until normal breathing resumed. A death rate of $33 \%$ was observed for injured animals. Righting reflex time was recorded for each animal, averaging 34 minutes (range of 17 to 60 minutes). Injury severity was determined based on functional behavior as measured by the neurological severity score (NSS), assessed at 24 hours after injury. In total 8 animals received the insult (TBI Group) and 11 animals received only the craniectomy (Control Group).

\section{Diffusion Tensor Imaging}

To record changes in brain network structure resulting from TBI, Diffusion Tensor Imaging (DTI) scans were performed 5 weeks after the insult with a $20 \mathrm{~cm}$ diameter horizontal- 
bore 9.4 Tesla magnet (Biospec 94/20 USR, Bruker Biospin) equipped with imaging gradient coils supporting $440 \mathrm{mT} / \mathrm{m}$ gradient strength and $3440 \mathrm{~T} / \mathrm{m} / \mathrm{s}$ maximum linear slew rate (BGA12S HP; Bruker Biospin). We employed a cross-coil configuration with a 4-channel receiver ratbrain surface array and a transmitter volume coil. The scanner was operated with ParaVision (version 5.1; Bruker Biospin). The DTI scans consisted of a respiration-gated 3D Spin-Echo DTI sequence with a two-shot echo-planar readout and reversed readout gradients for Nyquist ghost suppression. DTI acquisition parameters were as follows: $213 \times 144 \times 166 \mu \mathrm{m}^{3}$ field of view; 133x90x52 matrix zero-filled to $266 \times 180 \times 104 ; 1$ average; TE/TR $25.96 \mathrm{~ms} / 1000 \mathrm{~ms}$; $B=1000$ $\mathrm{s} / \mathrm{mm} 2$ in 30 directions across the whole sphere; 4 unweighted acquisitions (B0); 3ms diffusion times at $415.9 \mathrm{mT} / \mathrm{m}$ and $12 \mathrm{~ms}$ duration. The acquisition time with gating was approximately 90 to 120 minutes.

\section{DTI Preprocessing and Structural Connectivity}

Voxel dimensions of the diffusion images were scaled by a factor of 10x. Motion and eddy-currents were corrected with FSL Eddy (Andersson and Sotiropoulos, 2016). Post eddy and motion correction, B0 images were averaged to create a brain mask using FSL Brain Extraction Tool (BET) (Smith, 2002). Advanced Normalization Tools (ANTs) (Avants et al., 2008), specifically ANTs Syn with default parameters, was used to register a brain atlas with 150 regions spanning cortical and subcortical areas (Valdés-Hernández, 2011) to the average of the BOs.

\section{Fiber Tracking and Network Construction}

Fiber tracking was done in DSI Studio with a modified FACT algorithm (Yeh et al., 2013). Whole brain seeding was used to generate 500,000 tracts with a fractional anisotropy threshold of 0.12 and angular threshold of $45^{\circ}$, step size of $1 \mathrm{~mm}$, minimum length of $2 \mathrm{~mm}$, maximum 
length of $300 \mathrm{~mm}$ based on the scaled voxel dimensions. In order to fully sample the fiber orientations within a voxel, tracking was repeated 1,000x with initiation at a random sub-voxel position generating 1,000 connectivity matrices per animal.

For each animal, an undirected weighted structural connectivity matrix, A, containing 150 brain regions was constructed by counting the number of streamlines between brain regions. The final connectivity matrix based on the average of the 1000 matrices. Connectivity matrices were then normalized by dividing the number of streamlines $(T)$ between region $i$ and $j$, by the combined volumes $(v)$ of region $i$ and $j$.

$$
A_{i j}=\frac{T_{i j}}{v_{i}+v_{j}}
$$

\section{Network Similarity}

We assessed global dis-similarity between two subjects, $p$ and $q$, based on the similarity of their structural connectivity using the Euclidean distance. Euclidean distance is defined as,

$$
D_{p q}=\sqrt{\sum\left(A_{p}-A_{q}\right)^{2}}
$$

where $A_{p}$ and $A_{q}$ correspond to the structural connectivity matrices from subject $p$ and $q$, respectively.

\section{Eigenvalue Spectrum}

Each connectivity matrix $(A)$ was decomposed to corresponding eigenvalues and eigenvectors. Eigendecomposition is obtained by solving the linear equation,

$$
A v=\lambda v
$$

where $\lambda=\left[\begin{array}{lll}\lambda_{1} & \ldots & \lambda_{n}\end{array}\right]$ are the corresponding eigenvalues (EigVal) and $v=\left[v_{1} \ldots v_{n}\right]$ the eigenvectors of $A$. The eigenvalue spectrum of $A$ describes structural properties of the network. 
Statistical differences were based on the distribution of eigenvalues as assessed with the Kolmogorov-Smirnov (K-S) test between the pooled TBI and Controls. We report the p-value and K-S test statistic (D) with a range $[0,1]$ which quantifies the maximum differences between the cumulative distributions.

\section{Network Based Statistics}

Network-Based Statistics (NBS) (Zalesky et al., 2010) was used to identify subnetworks which increased or decreased in strength due to trauma. NBS implementation involves initially calculating the T-statistic for each connection between animals in the TBI and Control groups followed by applying a primary threshold to the T-statistic to identify a subnetwork. The threshold results in subnetwork with size $L$, where size corresponds to the number of connections in the subnetwork. Statistical significance of the subnetwork is based on randomizing the group labels between individuals and calculating the size of the subnetwork after randomization. Familywise error (FWE)-corrected $p$ values were calculated on the subnetwork using a null distribution derived from 5,000 permutations. Subnetworks that survived a network-level threshold of $p<0.05$ (FWE corrected) were considered significant.

Since the choice of a primary T-statistic threshold is arbitrary, we ran the analysis over a range of T-statistical values from 1 to 2.5 in steps of 0.1 and further analysis was based on the T-statistic threshold which minimized the corrected $p$-value. For the subnetwork that increased in strength in the TBI group, analytical results are presented for T-threshold of 1.8. For the subnetwork that decreased in strength in TBI group, analytical results are presented for Tthreshold of 2.0

\section{Minimum Spanning Tree}


The Minimum Spanning Tree (MST) is a subnetwork that connects all nodes while minimizing edge weights without forming loops (Kruskal, 1957; Tewarie et al., 2015). Since in brain networks, edge weights represent connectivity strength between regions, we calculated the MST using the inverse of the connections in structural connectivity. We additionally calculated the cost network associated with the MST (MST-Cost). Network cost is defined as the connection weight multiplied by the Euclidian distance between the centroids of two regions (Achard et al., 2006; Heuvel et al., 2012).

Statistical differences were based on the distribution of connections assessed with the Kolmogorov-Smirnov (K-S) test between the pooled TBI and Controls. We report the p-value and K-S test statistic (D) with a range $[0,1]$ which quantifies the maximum differences between the cumulative distributions.

\section{Motif Analysis}

Motifs $(M)$ are patterns of network connections among a specified number of nodes forming a subgraph $(g)$, which act as building blocks for complex networks (Sporns and Kötter, 2004). Given that our structural connectivity matrices are undirected, our analysis focused on two types of three-node motifs and six types of four-node motifs. Individual motifs are named using the convention from Sporns and Kötter, 2004.

To assess how strongly a brain region participates in a specific motif, we calculated the motif coherence $(Q)$ for each brain region (Onnela et al., 2005). We used motif coherence because it accounts for low probability values due to one connection weight being low versus all connection weights being low (Onnela et al., 2005). For each individual motif pattern, $g, Q$ is defined as

$$
Q_{g}=\frac{\left|l_{g}\right|\left(\prod_{(i j) \in g} A_{i j}\right)^{1 /|l g|}}{\sum_{(i j) \in g} A_{i j}}
$$


where $l_{g}$ is the set of links in the specified subgraph, $\left|l_{g}\right|$ is the number of links in that subgraph, and $A$ is the connection strength between brain region $i$ and $j$. Motif coherence was calculated using the Brain Connectivity Toolbox (Rubinov and Sporns, 2010).

To assess changes in motif coherence for each brain region, statistical significance was based on the difference in the TBI and subgroups when compared to the Control group. Specifically,

$$
Q_{D}=<Q_{T}>-<Q_{C}>
$$

where $Q_{D}$ is the difference between the $\left\langle Q_{T}>\right.$ and $\left\langle Q_{C}>\right.$ representing the average motif coherence for the subgroups or TBI and Control groups, respectively. Statistical significance was based on permuting group labels $10,000 x$ and brain regions with motif Z-scores $\left(Z_{Q}\right)$ greater or lower than 1.96 from the null distribution (corresponding to p-values of 0.05 or lower) were considered statistically significant. Specifically, for each motif subtype, $Z_{Q}$ was calculated as,

$$
Z_{Q}=\frac{Q_{D}-<Q_{D_{\text {Null }}}>}{\sigma_{D_{\text {Null }}}}
$$

where $<Q_{D_{N u l l}}>$ and $\sigma_{D_{N u l l}}$ represent the average and standard deviation of the coherence for a motif subtype across the 10,000 permutations, respectively. For plotting purposes, we average the Z-scores of a node across all statically significant motif subtypes.

\section{Clustering Analysis}

To test for the existence of subpopulations, we designed a two-step unsupervised clustering procedure that utilized network measures to cluster animals into subgroups. In the first step, using kmeans.m in MATLAB, we clustered individuals independently across 13 network measures: Structural Connectivity (SC), Eigenvalue spectrum (EigVal), NBS, MST, MST-Cost, and 8 distinct Motifs. Similarity between animals was calculated using the L1-Norm. 
The optimal number of clusters $(K)$ was determined by iterating $K$ from 1 to $\mathrm{N}\left(\mathrm{N}_{\mathrm{TBI}}=8\right.$; $\mathrm{N}_{\text {Control }}=$ 11 , respectively) and the $K$ with the maximum average silhouette value, which measures how similar a subject is to other subjects in the same cluster, was chosen as the optimal number of clusters.

In the second step, we created, an association matrix based on the degree of similarity between animals calculated as the number of times two animals were in the same cluster across the 13 network measures. Subgroups within a population were identified by clustering the association matrix using modularity-maximization. Modularity-maximization identifies communities in which the similarity within a cluster is greater than between clusters. In addition, modularity-maximization does not require a predefined number of clusters to be specified. The resolution of the clusters can be controlled with parameter, $\mathrm{\gamma}$.

Modularity-maximization was implemented with the Generalized Louvain algorithm $(\mathrm{Y}=$ 1) (Mucha et al., 2010). In order to avoid such suboptimal results due to initial seeding, clustering was repeated $100 x$ and final results were based on the consensus across the 100 runs (Bassett et al., 2013).

\section{Predicted Functional Connectivity}

Functional connectivity was predicted using the path transitivity (Goni et al., 2014). Path transitivity estimates the amount of signal that propagates from brain region $i$ to $j$, while taking into account the number or re-entrants connections along the shortest path (AvenaKoenigsberger et al., 2017). Path Transitivity was calculated using the Brain Connectivity Toolbox (Rubinov and Sporns, 2010).

To assess changes in predicted functional connectivity, statistical significance was based on the difference in the TBI and subgroups when compared to the Control group. We first calculated the average predicted functional connectivity, $\left\langle F C_{T / S}\right\rangle$, for the $T B I$ and for the 
subgroups followed by subtracting the average predicted functional connectivity from the Control, $<F C_{C}>$.

$$
F C_{D}=<F C_{T / S}>-<F C_{C}>
$$

The resulting values represent the overall change in predicted functional connectivity $F C_{D}$, for the $\mathrm{TBI}$ and subgroups, respectively. We then assessed differences between the TBI and the subgroups with using an Analysis of Variance (ANOVA).

However, because this ANOVA does not assess which specific connections have a higher propensity to change, we performed additional analysis. To assess changes in individual connections, statistical significance was based on permuting group labels 10,000x and connections with Z-scores $\left(Z_{F C}\right)$ greater or lower than 1.96 from the null distribution (corresponding to p-values of 0.05 or lower) were considered statistically significant. Specifically, for each connection, $Z_{F C}$ was calculated as

$$
Z_{F C}=\frac{F C_{D}-<F C_{D_{\text {Null }}}>}{\sigma_{D_{\text {Null }}}}
$$

$<F C_{D_{N u l l}}>$ and $\sigma_{D_{N u l l}}$ represent the average and standard deviation of the predicted functional connectivity across the 10,000 permutations, respectively.

\section{Experimental design and statistical analysis}

The lateral fluid percussion model of TBI was used to induce a severe injury in Wistar rats $(n=8)$ and sham controls $(n=11)$ (Figure 1 Left). The sham controls received the surgery, but no injury. Five weeks after the injury, animals underwent DTI imaging (Figure 1 Middle) from which tractography and structural connectivity was determined (Figure 1 Right).

Network measures were calculated using the Brain Connectivity Toolbox (Rubinov and Sporns, 2010) and unique scripts in MATLAB 2017B (MathWorks). Statistical analyses were 
based on a combination of parametric and nonparametric tests, see individual methods sections for details. In addition, BrainNet Viewer was used to display results (Xia et al., 2013).

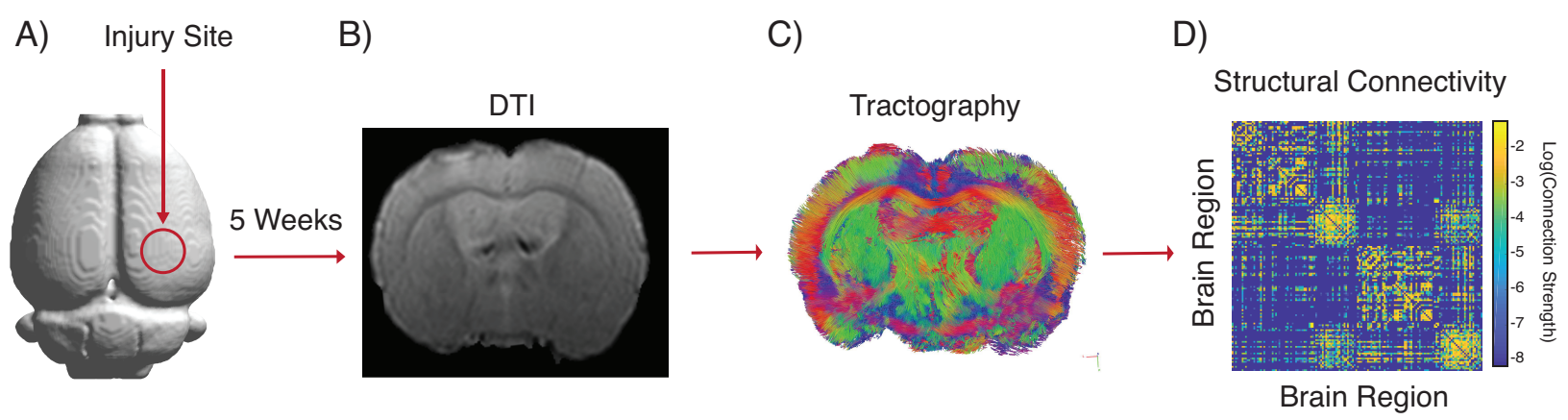

Figure 1. Experimental design and analysis. A) Injury was induced in the right hemisphere using the lateral fluid percussion model. B) Five weeks after injury animals underwent DTI imaging from which (C) white matter tracts and (D) structural connectivity was derived.

\section{Results}

Here we quantify changes in structural connectivity in a population of 8 animals that received a severe-TBI using the lateral-fluid percussion injury (FPI) model and 11 control animals that received only the craniectomy surgery. Five weeks after the injury, animals underwent DTI imaging from which structural brain networks were created.

\section{Between Group Similarity}

We first assessed changes in brain network structure occurring at the global level in both TBI and Control animals in an attempt to identify types of large-scale structural changes associated with TBI. Specifically, we used the Euclidean distance between structural connectivity matrices to measure the magnitude of dis-similarity between the TBI and the Control group (Figure 2A). Comparing the degree of dis-similarity within a group (Within-Grp: $0.36 \pm 0.035(S D)$ ) to between groups (Between-Grp: $0.37 \pm 0.029(S D)$ ) we found no significant differences $\left(T_{169}=-0.73, p=0.46\right)$ (Figure $\left.2 B\right)$. We also examined the eigenvalue spectrum of 
the networks but observed no differences between the TBI and Control groups (KolmogorovSmirnov test; $D=0.016 ; p=0.99$ ) (Figure $2 C$ ). These results indicate that at the global level, measures of network structure are not able to distinguish between the two groups.

A)

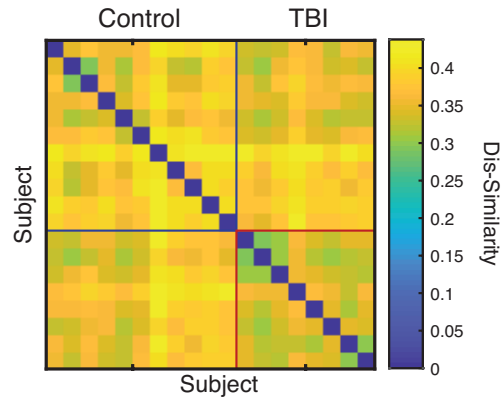

B)

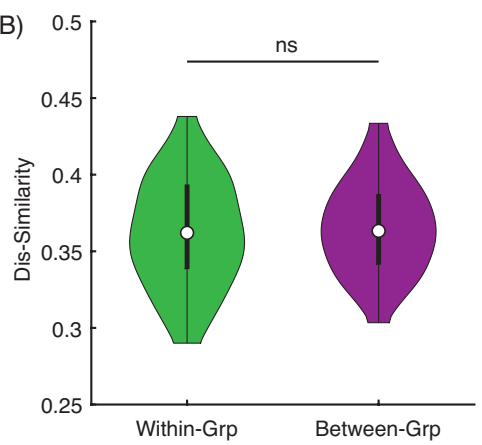

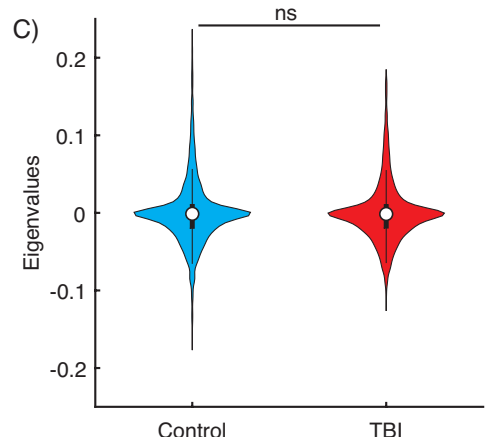

Figure 2. Global dis-similarity between groups. A) the magnitude of dis-similarity between all animals calculated using the Euclidian distance between structural connectivity networks. B) We observed no significant differences in the magnitude of Within- and Between-Group dissimilarity $\left(\mathrm{T}_{169}=-0.73, \mathrm{p}=0.46\right)$. C) The distributions of Eigenvalue spectrums for $\mathrm{TBI}$ and Control groups were not different (Kolmogorov-Smirnov test; $D=0.016 ; p=0.99$ ). For each violin plot, the central mark indicates the median, the bottom and top edges of the box indicate the 25th and 75th percentiles, respectively and whiskers extend to the most extreme data points not considered outliers.

\section{NBS and MST Analysis}

We next asked if the injury induced by the FPI primarily effects only a subset of connections in the brain. To accomplish this, we used Network Based Statistics (NBS) to identify subnetworks which increased or decreased in strength in the TBI group relative to the Control group. With NBS, we identified a subnetwork consisting of 268 connections ( $n=8$ rats), which increased in strength (Figure 3A) $(p<<0.001)$. In addition, the magnitude of the increase in strength of the connections was negatively correlated with the distance between brain regions $(r=-0.46, p=$ 
$\left.1.53 \times 10^{-15}\right)$ (Figure 3B). Alongside this component, NBS identified a smaller subnetwork with 74 connections ( $n=8$ rats) in which the connection strengths decreased (Figure $3 C)(p<<$ $0.001)$.

A)
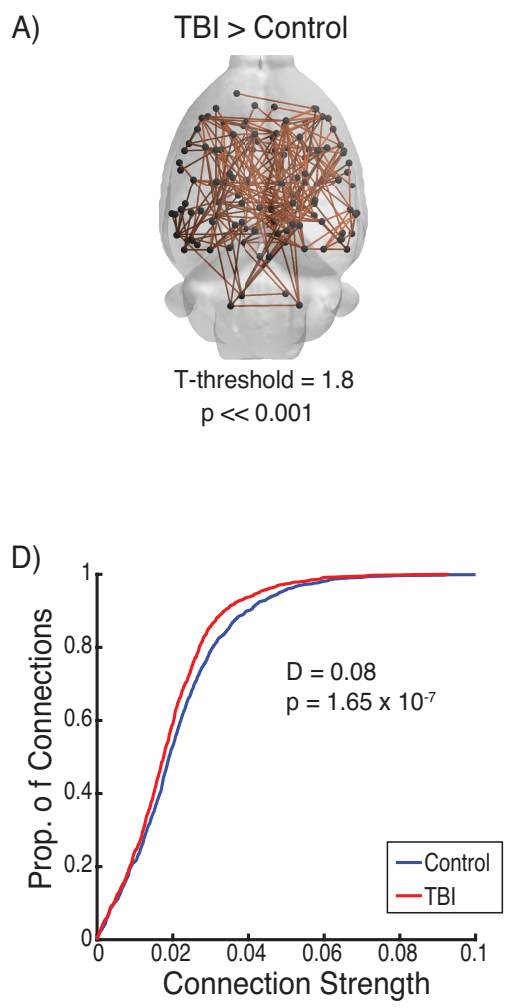

B)

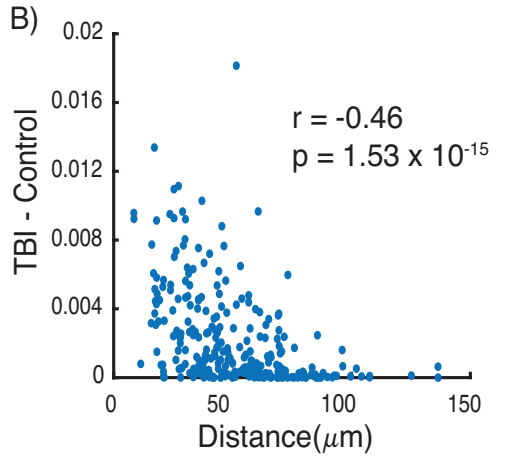

E)

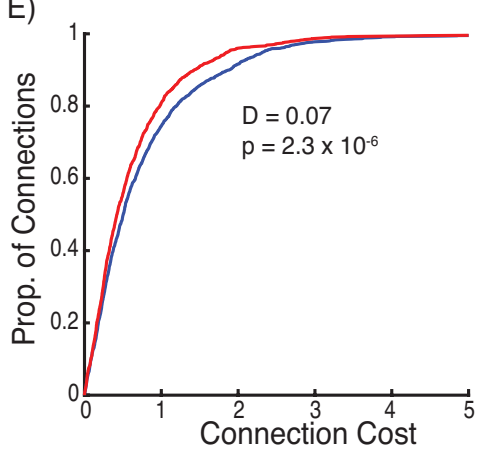

C)
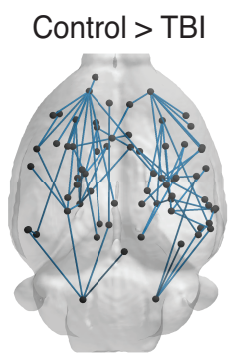

T-threshold $=2.0$ $\mathrm{p} \ll 0.001$

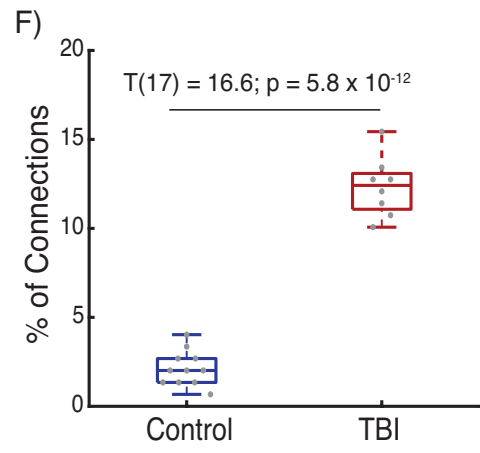

Figure 3. Injury induced changes in structural connectivity subnetworks. A) NBS identified a subnetwork increasing in connectivity strength in the TBI group. B) Correlation of magnitude differences of the subnetwork from $(A)$ with distance between brain regions $(r=-0.46, p=1.53 x$ $10^{-15}$ ). C) Subnetwork of connections found with NBS that decreased in strengths in the TBI group. D and E) Differences in the proportion of connections in the TBI and Controls for the MST- $\left(D=0.08, p=1.65 \times 10^{-7}\right)$ and MST-Cost subnetworks $\left(D=0.07, p=2.3 \times 10^{-6}\right)$, respectively. F) Percentage of connections in the MST that are present in the NBS subnetwork for TBI and Controls $\left(\mathrm{T}_{17}=16.6, \mathrm{p}=5.8 \times 10^{-12}\right)$. For each boxplot, the central mark indicates the median, the bottom and top edges of the box indicate the 25th and 75th percentiles, 
respectively and whiskers extend to the most extreme data points not considered outliers.

We also assessed if there were corresponding changes in the network backbone as measured with the minimum spanning tree (MST). We found that TBI induced a shift in the MST toward weaker $\left(\mathrm{MST}: \mathrm{D}=0.08, \mathrm{p}=1.65 \times 10^{-7}\right)$ and lower cost connections (MST-Cost: $\mathrm{D}=$ $\left.0.07, p=2.3 \times 10^{-6}\right)$ (Figure 3D and $E$, respectively). Lastly, we assessed for an association between connections found in the NBS and MST. We found significant differences in the percentage of connections in common between the NBS and MST subnetworks between the two groups $\left(\mathrm{T}_{17}=16.6, \mathrm{p}=5.8 \times 10^{-12}\right)($ Figure $3 \mathrm{~F})$. Specifically, in the TBI group $12.3 \% \pm 1.68$ (SD) of connections were present in both subnetworks, while the Control group only $2.1 \% \pm$ 0.98 (SD) were present in both subnetworks.

\section{Motif Analysis}

Our initial analysis identified a series of changes in subnetwork network structure that differed between TBI and Control groups, indicating that injury induced changes can be observed at the local network level. We followed up these observations with a further assessment of local connectivity and investigated the changes that TBI induces in motif coherence, which measures how strongly a brain region participates in specific patterns of connections (Materials and Methods; Figure 4A) (Onnela et al., 2005). In the TBI group, 9.3\% of brain regions ( $N=14$ brain regions from $n=8$ rats) increased in motif coherence, and $6 \%$ of brain regions ( $\mathrm{N}=9$ brain regions from $\mathrm{n}=8$ rats) decreased in motif coherence relative to the Control group (Figure 4B). Statistical significance was based on a permutation test in which group labels were permutated 10,000x. See Materials and Methods for details. These results confirm that in the TBI group, we see changes in network structure occurring at the local level. 
A)

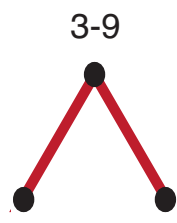

4-113

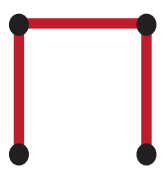

4-187

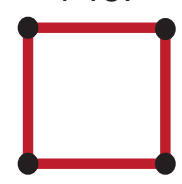

4-126

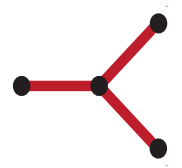

4-193

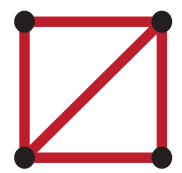

3-13

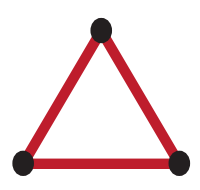

4-128

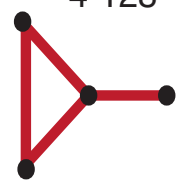

4-199

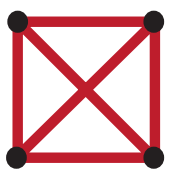

B)

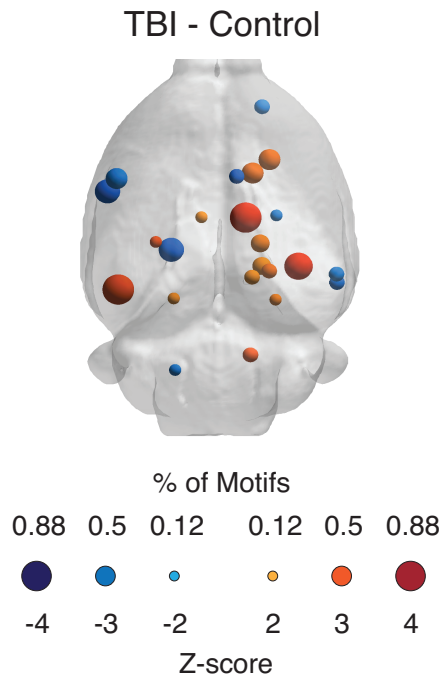

Figure 4. Motif analysis. A) Schematic of 3- and 4-node motifs. B) Regions for which differences in motif coherence are statistically significant in the TBI group. Color corresponds to the average Z-score of all significant motifs and size corresponds to percentage of motifs which a brain region was significantly different.

\section{Clustering Analysis}

Thus far, our analysis of subnetworks and local structure has focused on examining differences between TBI and Control rats. However, one of the hallmarks of TBI is the diverse response in patients (Sidaros et al., 2008; Kinnunen et al., 2011; Ghajari et al., 2017) with a subset of patients and animals developing post-traumatic epilepsy (Frey, 2003; Kharatishvili et al., 2006). This diverse response implies the existence of subpopulations within the TBI group due to different trajectories in pathological progression. To test for the existence of subpopulations within the TBI group, we designed a two-step unsupervised clustering procedure that utilized network measures to test for the presence of subgroups as described in the Materials and Methods. Clustering based on individual network measures resulted in the detection of 2 clusters in the TBI group with the exception of Motif 4-199 which contained 3 clusters (individual columns of Figure 5A). Using these results to perform our final clustering 
step, we identified two subgroups in the TBI population, which we labeled T1 ( $n=3$ rats), and T2 ( $n=5$ rats) subgroup (Figure 5B). In addition, the motifs were the primary network measures that separated the TBI animals into subgroups (Figure $5 \mathrm{~A}$ ).
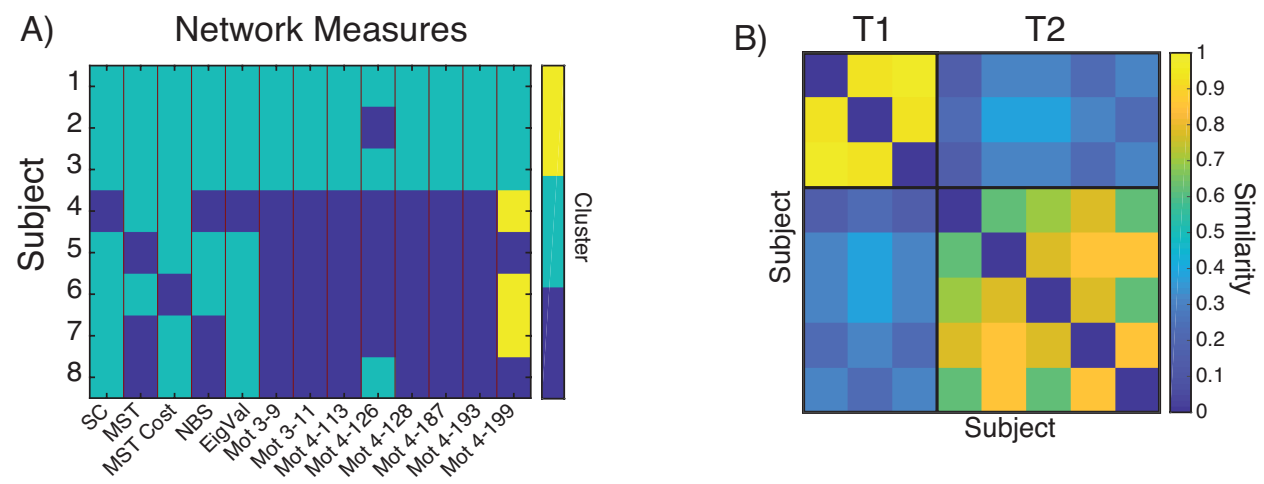

Figure 5. Clustering analysis and subpopulation identification of animals with TBI. A) K-means was used to cluster each animal within each network measures. Columns represent the cluster identity of each animal clustered with a network property. The color represents a unique cluster identity. B) Modularity-maximization clustering on cluster labels from (A) was used to identify two subpopulations, T1 and T2, in the TBI group. Similarity is based on the fraction of network properties that two rats have the same cluster identify from (A).

When the same analysis was performed on the Control group, we also identified two subpopulations (Figure 6A and B). However, as can be clearly seen in Figure 6B, the within vs between similarities are nearly identical between these two subgroups. Additionally, the between subgroup similarity was significantly greater $\left(T_{31}=4.4, p=1.18 \times 10^{-4}\right)$ in the Controls $(75 \% \pm 21(\mathrm{SD}))$ than the TBI $(28 \% \pm 0.09(\mathrm{SD}))$ (Figure 6C). This suggests that, despite the algorithm identifying two subpopulations in the Control group, the presence of these subgroups in the Control group is an artifact of the clustering algorithm and not indicative of true between subgroup differences as were observed in the TBI group. 

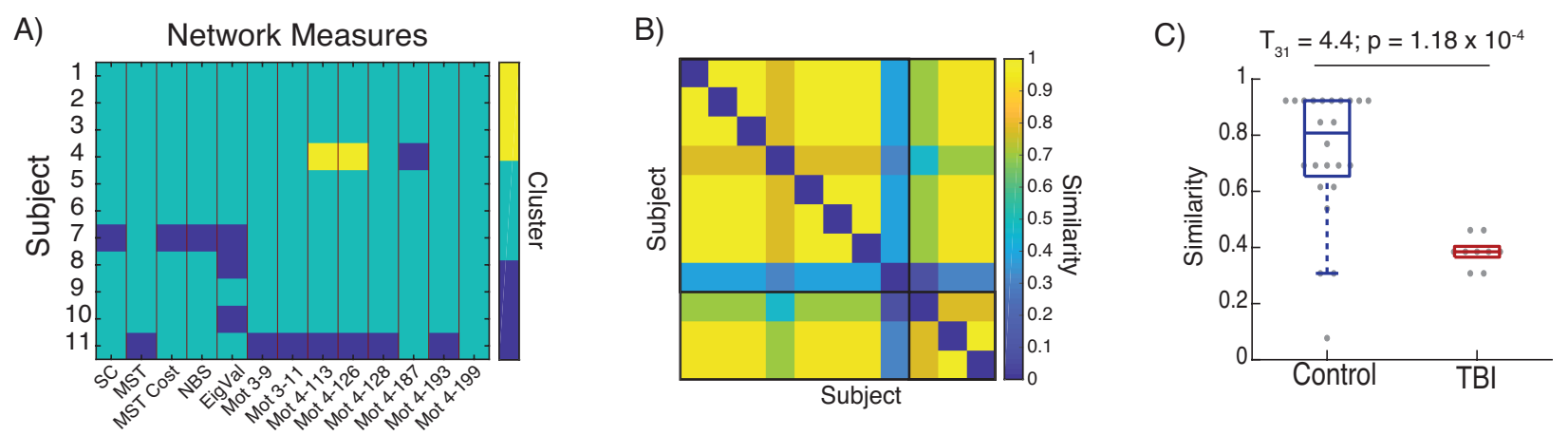

Figure 6. Clustering analysis of controls animals. A) K-means clustering was used to cluster each animal within each network measure. Columns represent the cluster identity of each animal clustered with a network property. The color represents a unique cluster identity. B) Modularity-maximization clustering on cluster labels form (A). C) Magnitude of the similarity between identified subpopulations in TBI and Controls $\left(T_{31}=4.4, p=1.18 \times 10^{-4}\right)$. For each boxplot, the central mark indicates the median, the bottom and top edges of the box indicate the 25th and 75th percentiles, respectively and whiskers extend to the most extreme data points not considered outliers.

\section{Subgroup Motif Analysis}

Our analysis indicated that the TBI population separates into two subgroups, and as can be seen if Figure 5A, this separation is driven by alterations in the expression of structural connectivity motifs. To gain a better understanding of these changes, we analyzed how motif expression changed within the TBI animals within each subgroup. Specifically, we compared the changes in motif coherence between the T1- and T2-subgroups relative to those of the Control group. To account for the low number of animals, statistical testing was based on a permutation test in which subgroup labels were permutated 10,000x.

In the T1-subgroup, $38 \%$ of brain regions $(\mathrm{N}=57$ brain regions from $\mathrm{n}=3$ rats) increased in their motif coherence, while $0.7 \%$ of brain regions $(\mathrm{N}=1$ brain regions from $\mathrm{n}=3$ 
rats) decreased in their motif coherence relative to Controls (Figure 7A). In contrast, in the T2subgroup, only $3.3 \%$ of brain regions ( $N=5$ brain regions from $n=5$ rats) increased in their motif coherence, while $15.3 \%$ of brain regions ( $N=23$ brain regions from $n=5$ rats) decreased their in motif coherence relative to Controls (Figure 7B). Thus, in the T1-subgroup, structural reorganization causes brain regions to increase their participation in local motifs, while in the T2-subgroup, the reorganization results in brain regions decreasing their participation in motifs.

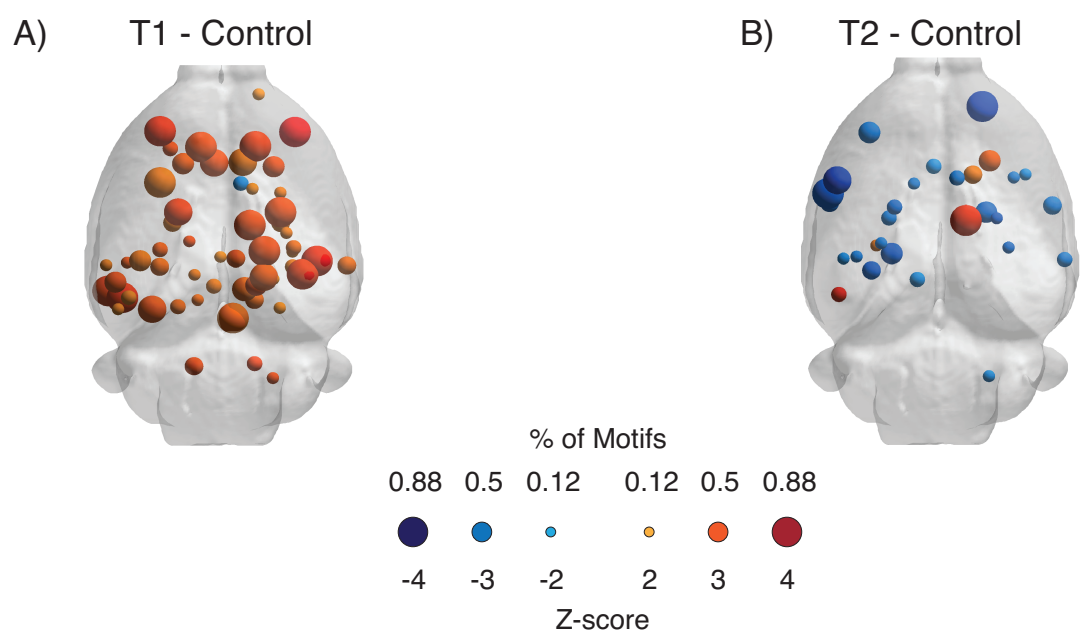

Figure 7. Regions for which differences in motif coherence are statistically significant. A) T1and B) T2-subgroups for which a brain region's motif coherence was significantly different from Controls. Color corresponds to the average Z-score of all significant motifs and size corresponds to percentage of motifs which a brain region was significantly different.

\section{Predicted Functional Connectivity}

Given that we observed two subgroups with different types of structural changes in TBI animals, we next asked if these structural differences might underlie differences in functional brain activity between the two subgroups, specifically with the propensity of each of these subgroups to promote synchronous brain activity (Terry et al., 2012). Functional connectivity describes statistical relationships between the activity of two brain regions, and the degree of 
functional connectivity can be associated with synchronization in brain activity. To assess potential differences in synchronized activity between the two subgroups, we tested if the structural differences observed between the T1- and T2-subgroups might result in altered functional connectivity. To predict functional activity of the two subgroups, we used a computational approach based on path transitivity (Goni et al., 2014) (see Materials and Methods).

In Figure 8A, we show the average predicted functional connectivity for the Control group, T1, and T2 subgroups. We first used an ANOVA to assess average change in predicted functional connectivity. We found that the average change in functional connectivity was significantly different $\left(F_{2,32631}=1758, p<0.0001\right)$ between all TBI (TBI-Control: $0.008 \pm 0.04$ (SD)), T1-subgroup (T1-Control: $0.035 \pm 0.06(\mathrm{SD})$ ) and T2-subgroup (T2-Control: $-0.008 \pm 0.05$ (SD)) relative to controls (Figure $8 \mathrm{~B}$ ). We next examined changes in individual connections in predicted functional connectivity and found that when compared to the Control group, in the TBI group $5.6 \%$ of connections increased $(N=630$ edges in $n=8$ rats $)$ in strength, while $1.9 \%$ of connections decreased ( $N=207$ edges in $n=8$ rats) in strength (Figure $8 C$ ). When separating the animals into their respective subgroups, the T1-subgroup showed $9.2 \%$ of connections $(\mathrm{N}=$ 1033 edges in $n=3$ rats) increased and $0.7 \%$ of connections decreased $(N=81$ edges in $n=3$ rats) in strength (Figure 8D). In contrast, the T2-subgroup showed $3.8 \%$ of connections $(\mathrm{N}=$ 422 edges in $n=5$ rats $)$ increased and $4.3 \%$ of connections decreased $(N=483$ edges in $n=5$ rats) in strength (Figure 8E). Interestingly, in both the T1- and T2- subgroups the connections that decreased in strength were located adjacent to the site of the injury. 
A)

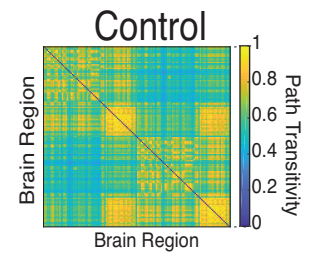

C)

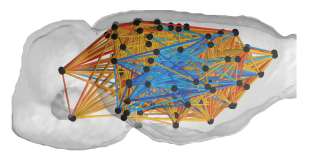

D)

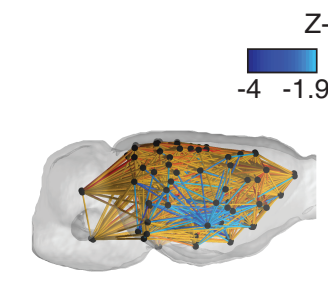

E)

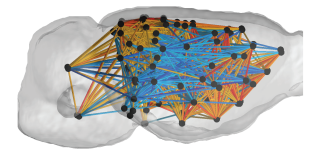

Right

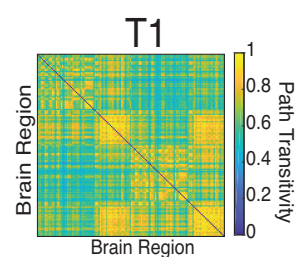

TBI - Control
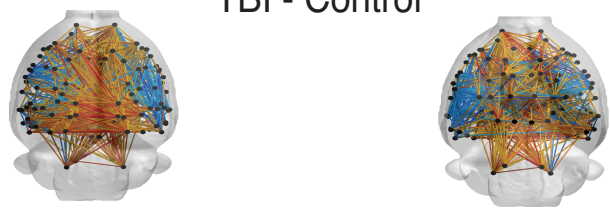

T1 - Control

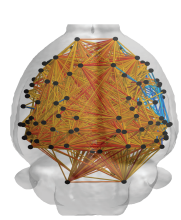

T2 - Control
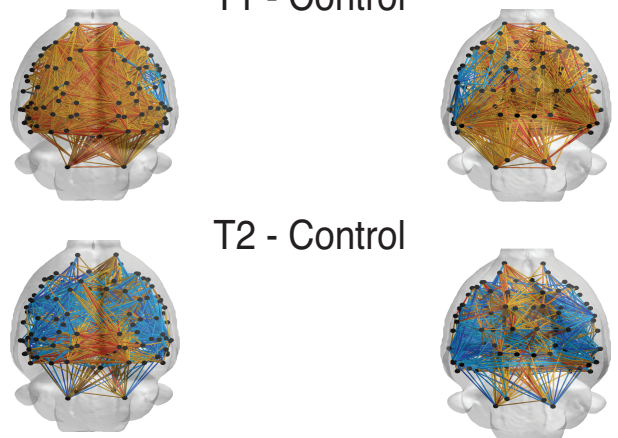

Top

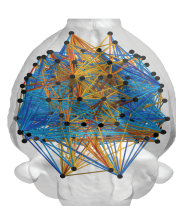

Bottom
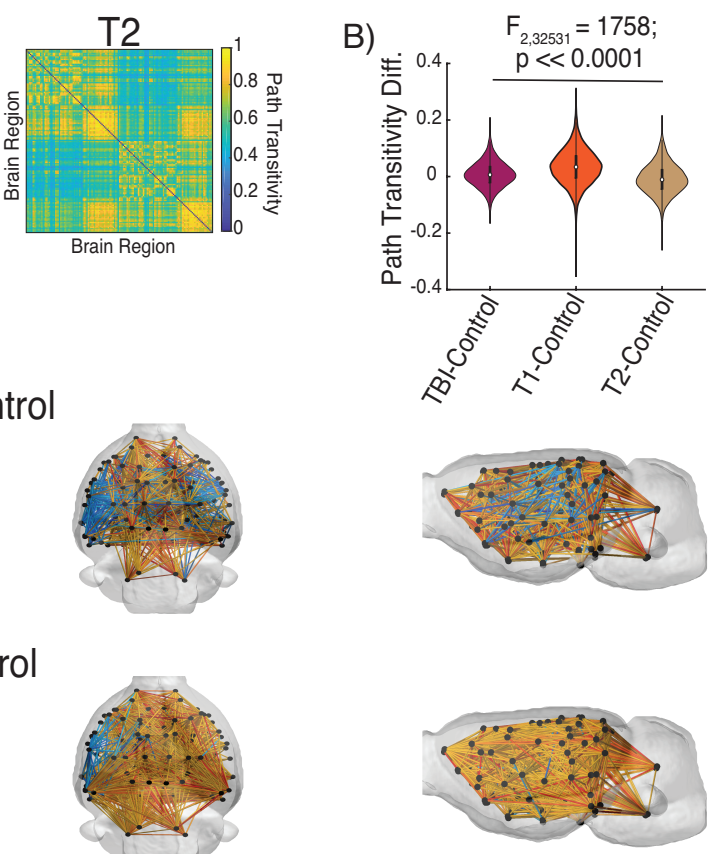

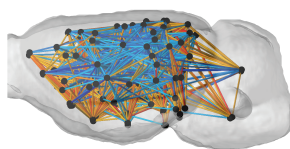

Left

Figure 8. Predicted and changes in functional connectivity between TBI subgroups and Control group. A) Average predicted functional connectivity for the Control Group (Left), T1- (Middle) and T2- (Right) subgroups. B) Changes in average predicted functional connectivity between TBI, T1- and T2-subgroup. C-E) Individual connections which were significantly different in the TBI group, T1- and T2-subgroup from the Control groups, respectively.

\section{Discussion}

TBI is marked by focal and diffuse jury to both grey and white matter in the brain (Gentleman et al., 1995; Smith et al., 2003; Johnson et al., 2013b). Along with structural damage, TBI has been associated with a variety of adverse functional/behavioral changes. For example, TBI increases the risk of developing post-traumatic epilepsy (Annegers et al., 1998; 
Englander et al., 2003; Christensen et al., 2009; Wang et al., 2013; Mahler et al., 2015; Webb et al., 2015; Smith et al., 2018) and it has been shown that a subpopulation of TBI patients with a similar pattern of abnormal network connections exhibited decreased cognitive performance (Solmaz et al., 2017). Currently no clear biomarkers exist for predicting who will and will not develop adverse functional effects, so it is important to characterize the heterogeneity of brain structural changes resulting from injury in order to relate those changes to functional outcomes (Pitkänen and Immonen, 2014). Here we use network theory to characterize changes in global and local connectivity following TBI in rats induced by FPI in order to probe how heterogeneity in structural changes might drive some of the heterogeneity observed functionally and behaviorally.

We found that global measures of structural connectivity do not distinguish the TBI group from the Control group, despite animals in the TBI group receiving a severe injury, which was validated by functional assessment (NSS). Instead we found changes in a subset of connections and nodes which are consistent with previous work that found changes in the density for a subset of fibers (Harris et al., 2016a; Iraji et al., 2016; Wright et al., 2017), and subnetworks in structural connectivity following TBI (Hayes et al., 2016; Iraji et al., 2016; Thengone et al., 2016; Dall'Acqua et al., 2017). These alterations indicate adaptive and maladaptive changes in brain connectivity (Fornito et al., 2015). Adaptive responses could indicate myelin repair (Armstrong et al., 2016) and/or axonal sprouting post TBI (Christman et al., 1997; Harris et al., 2010). On the other hand, maladaptive changes could be a result of axonal death (Gentleman et al., 1995; Smith et al., 2003), myelin damage (Bramlett and Dietrich, 2002; Johnson et al., 2013b) and neuroinflammation (Johnson et al., 2013a).

The changes in local connectivity structure were primarily found in shorter distance connections which increased in strength causing the network backbone to include more short range connections. These shifts in the network backbone could be indicative of a break down in 
efficient communication between brain regions (Avena-Koenigsberger et al., 2017). In addition to group level differences in subnetworks and local structure between injured and non-injured subjects, TBI has been found to induce subject-specific alterations in the underlying white matter raising the possibility of individualized pathological trajectories (Ware et al., 2017). In our analysis, we identified a set of changes in brain structure that could separate animals in the TBI group into two subpopulations, which we called the T1- and T2-subgroups. Our identification of subpopulations aligns with previous work in humans in which alteration in structural connectivity were able cluster individuals with differences extending to cognitive performance (Solmaz et al., 2017).

The fact that the presence of subgroups was driven by differences in motif participation indicates that local connections between brain regions could play an important role in network reorganization post-injury. Modeling studies that have focused on the relationship between motifs and synchronized activity have found that certain structural connectivity patterns, linear connections, Motif 3-9 (Figure 1), promote zero-phase lag synchrony (Gollo and Breakspear, 2014). At the level of neuronal connectivity, computational modeling studies have shown that the presence of certain motifs relates to sustained neural activity (Bojanek et al., 2020). Additionally, recent modeling work identified the presence of superhubs - highly connected neurons that drive network activity through feedforward motifs - in epileptic networks (Hadjiabadi et al., 2021). Thus, the reorganization and increase of motif coherence in the T1 subgroup suggests that this subgroup would be expected to experience a change in brain activity, such as synchronization, as well.

The relationship between an increase motif coherence and synchronization, is supported by our predicted functional connectivity analysis in which we found that the T1 subgroup had an in increase in predicted functional connectivity relative to the Control group and the T2 subgroup. This finding corroborates previous analyses that have found that TBI increases 
functional connectivity in humans, (Mayer et al., 2011; Sharp et al., 2011; Hillary et al., 2014) and rats (Harris et al., 2016b). This is important given that individuals that sustain a severe TBI (sTBI) are at a 17 times greater risk of developing epilepsy (Annengers et al., 1998; Banerjee et al., 2009; Houser et al., 1990), and simulation models of epilepsy have found that focal, secondary- and primary-generalized seizure can arise from changes in network architecture (Terry et al., 2012) . We might speculate that the T1 subgroup could represent animals that are more likely to go on to develop PTE. However, the animals were not monitored using videoEEG to assess long term outcomes. Despite this limitation, our analysis shows that network measures can be used to identify meaningful differences within a population and offers an insight pertaining to the disease progression for an individual.

Because TBI is a complex and heterogeneous disorder, where similar insults can give rise to different behavioral outcomes, it is essential to develop new analytical approaches that are tailored to the heterogeneities present within the data. Most work that searches for biomarkers of PTE looks for correlations between a single observed variable and the development of PTE. However, our findings suggest that future work should examine and embrace heterogeneity within subject populations to more effectively link specific patterns of brain reorganization to functional outcomes. 


\section{Author Contributions}

J.N. designed the analysis, processed the data, interpreted the data, and wrote/edited the manuscript.

M.G. designed the study, acquired data.

F.S. processed the data, acquired data, and edited the manuscript.

D.P designed the study, acquired data, and edited the manuscript.

S.F.M. designed the analysis, interpreted the data, and wrote/edited the manuscript.

\section{Conflict of Interest}

DP is a paid consultant for NeuroTrauma Sciences who provided funding for this study

\section{Funding Statement}

Research reported in this publication was funded in part by the National Center for Advancing Translational Sciences of the National Institutes of Health under Award Number UL1TR001412. This study was also funded in part by NeuroTrauma Sciences, as part of contract work order through the University at Buffalo. The content is solely the responsibility of the authors and does not necessarily represent the official views of the NIH or NeuroTrauma Sciences. 


\section{References}

Achard S, Salvador R, Whitcher B, et al. (2006) A Resilient, Low-Frequency, Small-World Human Brain Functional Network with Highly Connected Association Cortical Hubs. J Neurosci 26:63 LP - 72 Available at: http://www.jneurosci.org/content/26/1/63.abstract.

Andersson JLR, Sotiropoulos SN (2016) An integrated approach to correction for off-resonance effects and subject movement in diffusion MR imaging. Neuroimage 125:1063-1078.

Annegers JF, Hauser WA, Coan SP, et al. (1998) A Population-Based Study of Seizures after

Traumatic Brain Injuries. N Engl J Med 338:20-24 Available at:

https://doi.org/10.1056/NEJM199801013380104.

Armstrong RC, Mierzwa AJ, Marion CM, et al. (2016) White matter involvement after TBI: Clues to axon and myelin repair capacity. Exp Neurol 275:328-333.

Avants BB, Epstein CL, Grossman M, et al. (2008) Symmetric diffeomorphic image registration with cross-correlation: Evaluating automated labeling of elderly and neurodegenerative brain. Med Image Anal 12:26-41.

Avena-Koenigsberger A, Misic B, Sporns O (2017) Communication dynamics in complex brain networks. Nat Rev Neurosci 19:17-33.

Basser PJ, Mattiello J, LeBihan D (1994) MR diffusion tensor spectroscopy and imaging. Biophys J 66:259-267.

Bassett DS, Porter MA, Wymbs NF, et al. (2013) Robust detection of dynamic community structure in networks. Chaos.

Bassett DS, Sporns O (2017) Network neuroscience. Nat Neurosci 20:353-364 Available at: http://www.ncbi.nlm.nih.gov/pubmed/28230844\%0Ahttp://www.pubmedcentral.nih.gov/articl erender.fcgi?artid=PMC5485642.

Benson RR, Meda SA, Vasudevan S, et al. (2007) Global White Matter Analysis of Diffusion Tensor Images Is Predictive of Injury Severity in Traumatic Brain Injury. J Neurotrauma 
24:446-459.

Bojanek K, Zhu Y, MacLean J (2020) Cyclic transitions between higher order motifs underlie sustained asynchronous spiking in sparse recurrent networks. PLOS Comput Biol 16:1-30 Available at: https://doi.org/10.1371/journal.pcbi.1007409.

Bolkvadze T, Pitkänen A (2012) Development of Post-Traumatic Epilepsy after Controlled Cortical Impact and Lateral Fluid-Percussion-Induced Brain Injury in the Mouse. J Neurotrauma 29:789-812 Available at: https://doi.org/10.1089/neu.2011.1954.

Bramlett HM, Dietrich WD (2002) Quantitative structural changes in white and gray matter 1 year following traumatic brain injury in rats. Acta Neuropathol.

Budde MD, Janes L, Gold E, et al. (2011) The contribution of gliosis to diffusion tensor anisotropy and tractography following traumatic brain injury: Validation in the rat using Fourier analysis of stained tissue sections. Brain 134:2248-2260.

Caeyenberghs K, Leemans A, Leunissen I, et al. (2014) Altered structural networks and executive deficits in traumatic brain injury patients. Brain Struct Funct.

Christensen J, Pedersen MG, Pedersen CB, et al. (2009) Long-term risk of epilepsy after traumatic brain injury in children and young adults: a population-based cohort study. Lancet 373:1105-1110 Available at: https://www.sciencedirect.com/science/article/pii/S0140673609602142.

Christman CW, Salvant JB, Walker SA, et al. (1997) Characterization of a prolonged regenerative attempt by diffusely injured axons following traumatic brain injury in adult cat: A light and electron microscopic immunocytochemical study. Acta Neuropathol.

Dall'Acqua P, Johannes S, Mica L, et al. (2017) Functional and Structural Network Recovery after Mild Traumatic Brain Injury: A 1-Year Longitudinal Study. Front Hum Neurosci 11:116.

Englander J, Bushnik T, Duong TT, et al. (2003) Analyzing risk factors for late posttraumatic 
seizures: A prospective, multicenter investigation. Arch Phys Med Rehabil 84:365-373

Available at: https://www.sciencedirect.com/science/article/pii/S0003999302048402.

Fagerholm ED, Hellyer PJ, Scott G, et al. (2015) Disconnection of network hubs and cognitive impairment after traumatic brain injury. Brain 138:1696-1709.

Feldt S, Bonifazi P, Cossart R (2011) Dissecting functional connectivity of neuronal microcircuits: Experimental and theoretical insights. Trends Neurosci.

Fornito A, Zalesky A, Breakspear M (2015) The connectomics of brain disorders. Nat Rev Neurosci 16:159-172 Available at: http://dx.doi.org/10.1038/nrn3901.

Frey LC (2003) Epidemiology of Posttraumatic Epilepsy: A Critical Review. Epilepsia.

Gentleman SM, Roberts GW, Gennarelli TA, et al. (1995) Axonal injury: a universal consequence of fatal closed head injury? Acta Neuropathol.

Ghajari M, Hellyer PJ, Sharp DJ (2017) Computational modelling of traumatic brain injury predicts the location of chronic traumatic encephalopathy pathology. Brain 140:333-343.

Gollo LL, Breakspear M (2014) The frustrated brain: From dynamics on motifs to communities and networks. Philos Trans R Soc B Biol Sci 369.

Gollo LL, Mirasso C, Sporns O, et al. (2014) Mechanisms of Zero-Lag Synchronization in Cortical Motifs. PLoS Comput Biol 10.

Goni J, van den Heuvel MP, Avena-Koenigsberger A, et al. (2014) Resting-brain functional connectivity predicted by analytic measures of network communication. Proc Natl Acad Sci 111:833-838 Available at: http://www.pnas.org/cgi/doi/10.1073/pnas.1315529111.

Hadjiabadi D, Lovett-Barron M, Raikov IG, et al. (2021) Maximally selective single-cell target for circuit control in epilepsy models. Neuron 109:2556-2572.e6 Available at: https://www.sciencedirect.com/science/article/pii/S0896627321004220.

Harris NG, Mironova YA, Hovda DA, et al. (2010) Pericontusion axon sprouting is spatially and temporally consistent with a growth-permissive environment after traumatic brain injury. $\mathrm{J}$ 
Neuropathol Exp Neurol 69:139-154.

Harris NG, Verley DR, Gutman BA, et al. (2016a) Bi-directional changes in fractional anisotropy after experiment TBI: Disorganization and reorganization? Neuroimage 133:129-143 Available at: http://dx.doi.org/10.1016/j.neuroimage.2016.03.012.

Harris NG, Verley DR, Gutman BA, et al. (2016b) Disconnection and hyper-connectivity underlie reorganization after TBI: A rodent functional connectomic analysis. Exp Neurol 277:124138 Available at: http://dx.doi.org/10.1016/j.expneurol.2015.12.020.

Hayes JP, Bigler ED, Verfaellie M (2016) Traumatic brain injury as a disorder of brain connectivity. J Int Neuropsychol Soc 22:120-137.

Heuvel MP Van Den, Kahn RS, Goñi J, Sporns O, et al. (2012) High-cost, high-capacity backbone for global brain communication. Proc Natl Acad Sci U S A 109:11372-11377. Hillary FG, Rajtmajer SM, Roman CA, et al. (2014) The rich get richer: Brain injury elicits hyperconnectivity in core subnetworks. PLoS One 9.

Immonen R, Kharatishvili I, Gröhn O, et al. (2013) MRI Biomarkers for Post-Traumatic Epileptogenesis. J Neurotrauma 30:1305-1309 Available at: https://doi.org/10.1089/neu.2012.2815.

Iraji A, Chen H, Wiseman N, et al. (2016) Connectome-scale assessment of structural and functional connectivity in mild traumatic brain injury at the acute stage. Neurolmage Clin 12:100-115 Available at: http://dx.doi.org/10.1016/j.nicl.2016.06.012.

Johnson VE, Stewart JE, Begbie FD, et al. (2013a) Inflammation and white matter degeneration persist for years after a single traumatic brain injury. Brain.

Johnson VE, Stewart W, Smith DH (2013b) Axonal pathology in traumatic brain injury. Exp Neurol 246:35-43 Available at: http://dx.doi.org/10.1016/j.expneurol.2012.01.013.

Kharatishvili I, Nissinen JP, McIntosh TK, et al. (2006) A model of posttraumatic epilepsy induced by lateral fluid-percussion brain injury in rats. Neuroscience 140:685-697. 
Kinnunen KM, Greenwood R, Powell JH, et al. (2011) White matter damage and cognitive impairment after traumatic brain injury. Brain 134:449-463.

Königs M, van Heurn LWE, Bakx R, et al. (2017) The structural connectome of children with traumatic brain injury. Hum Brain Mapp 3614:3603-3614 Available at: http://doi.wiley.com/10.1002/hbm.23614.

Kraus MF, Susmaras T, Caughlin BP, et al. (2007) White matter integrity and cognition in chronic traumatic brain injury: A diffusion tensor imaging study. Brain 130:2508-2519.

Kruskal JB (1957) On the Shortest Spanning Subtree of a Graph and the Traveling Salesman Problem. Proc Am Math Soc 7:48-50.

Mac Donald CL, Dikranian K, Bayly P, et al. (2007) Diffusion Tensor Imaging Reliably Detects Experimental Traumatic Axonal Injury and Indicates Approximate Time of Injury. J Neurosci 27:11869-11876 Available at: http://www.jneurosci.org/cgi/doi/10.1523/JNEUROSCI.364707.2007.

Mahler B, Carlsson S, Andersson T, et al. (2015) Unprovoked seizures after traumatic brain injury: A population-based case-control study. Epilepsia 56:1438-1444 Available at: https://onlinelibrary.wiley.com/doi/abs/10.1111/epi.13096.

Mayer AR, Mannell MV, Ling J, et al. (2011) Functional connectivity in mild traumatic brain injury. Hum Brain Mapp 32:1825-1835.

McGuire MJ, Gertz SM, McCutcheon JD, et al. (2019) Use of a Wireless Video-EEG System to Monitor Epileptiform Discharges Following Lateral Fluid-Percussion Induced Traumatic Brain Injury. J Vis Exp:1-10.

McIntosh TK, Vink R, Noble L, et al. (1989) Traumatic brain injury in the rat: Characterization of a lateral fluid-percussion model. Neuroscience.

Mucha PJ, Richardson T, Macon K, et al. (2010) Community structure in time-dependent, multiscale, and multiplex networks. Science (80- ). 
Onnela JP, Saramäki J, Kertész J, et al. (2005) Intensity and coherence of motifs in weighted complex networks. Phys Rev E - Stat Nonlinear, Soft Matter Phys.

Pischiutta F, Micotti E, Hay JR, et al. (2018) Single severe traumatic brain injury produces progressive pathology with ongoing contralateral white matter damage one year after injury. Exp Neurol 300:167-178 Available at:

https://doi.org/10.1016/j.expneurol.2017.11.003.

Pitkänen A, Immonen R (2014) Epilepsy Related to Traumatic Brain Injury. Neurotherapeutics.

Rau TF, Kothiwal A, Rova A, et al. (2014) Phenoxybenzamine is neuroprotective in a rat model of severe traumatic brain injury. Int J Mol Sci.

Rau TF, Kothiwal AS, Rova AR, et al. (2012) Treatment with low-dose methamphetamine improves behavioral and cognitive function after severe traumatic brain injury. In: Journal of Trauma and Acute Care Surgery.

Rubinov M, Sporns O (2010) Complex network measures of brain connectivity: Uses and interpretations. Neuroimage 52:1059-1069 Available at:

http://dx.doi.org/10.1016/j.neuroimage.2009.10.003.

Sharp DJ, Beckmann CF, Greenwood R, et al. (2011) Default mode network functional and structural connectivity after traumatic brain injury. Brain 134:2233-2247.

Sharp DJ, Scott G, Leech R (2014) Network dysfunction after traumatic brain injury. Nat Rev Neurol 10:156-166 Available at: http://dx.doi.org/10.1038/nrneurol.2014.15.

Sidaros A, Engberg AW, Sidaros K, et al. (2008) Diffusion tensor imaging during recovery from severe traumatic brain injury and relation to clinical outcome: A longitudinal study. Brain 131:559-572.

Smith D, Rau T, Poulsen A, et al. (2018) Convulsive seizures and EEG spikes after lateral fluidpercussion injury in the rat. Epilepsy Res.

Smith DH, Meaney DF, Shull WH (2003) Diffuse axonal injury in head trauma. J Head Trauma 
Rehabil.

Smith SM (2002) Fast robust automated brain extraction. Hum Brain Mapp 17:143-155.

Solmaz B, Tunç B, Parker D, et al. (2017) Assessing connectivity related injury burden in diffuse traumatic brain injury. Hum Brain Mapp 38:2913-2922.

Sporns O, Kötter R (2004) Motifs in Brain Networks Karl J. Friston, ed. PLoS Biol 2:e369.

Terry JR, Benjamin O, Richardson MP (2012) Seizure generation: The role of nodes and networks. Epilepsia 53:166-169.

Tewarie P, van Dellen E, Hillebrand A, et al. (2015) The minimum spanning tree: An unbiased method for brain network analysis. Neuroimage.

Thengone DJ, Voss HU, Fridman EA, et al. (2016) Local changes in network structure contribute to late communication recovery after severe brain injury. Sci Transl Med 8.

Valdés-Hernández PA (2011) An in vivo MRI template set for morphometry, tissue segmentation, and fMRI localization in rats. Front Neuroinform 5:1-19 Available at: http://journal.frontiersin.org/article/10.3389/fninf.2011.00026/abstract.

van de Looij Y, Mauconduit F, Beaumont M, et al. (2012) Diffusion tensor imaging of diffuse axonal injury in a rat brain trauma model. NMR Biomed.

van der Horn HJ, Kok JG, de Koning ME, et al. (2017) Altered Wiring of the Human Structural Connectome in Adults with Mild Traumatic Brain Injury. J Neurotrauma 34:1035-1044 Available at: http://online.liebertpub.com/doi/10.1089/neu.2016.4659.

Wang H, Xin T, Sun X, et al. (2013) Post-traumatic seizures-A prospective, multicenter, large case study after head injury in China. Epilepsy Res 107:272-278 Available at: https://www.sciencedirect.com/science/article/pii/S092012111300260X.

Ware JB, Hart T, Whyte J, et al. (2017) Inter-Subject Variability of Axonal Injury in Diffuse Traumatic Brain Injury. J Neurotrauma 34:2243-2253 Available at: http://online.liebertpub.com/doi/10.1089/neu.2016.4817. 
Webb TS, Whitehead CR, Wells TS, et al. (2015) Neurologically-related sequelae associated with mild traumatic brain injury. Brain Inj 29:430-437 Available at: https://doi.org/10.3109/02699052.2014.989904.

Wilde EA, McCauley SR, Hunter JV., et al. (2008) Diffusion tensor imaging of acute mild traumatic brain injury in adolescents. Neurology.

Wright DK, Johnston LA, Kershaw J, et al. (2017) Changes in Apparent Fiber Density and Track-Weighted Imaging Metrics in White Matter following Experimental Traumatic Brain Injury. J Neurotrauma 34:2109-2118 Available at: http://online.liebertpub.com/doi/10.1089/neu.2016.4730.

Xia M, Wang J, He Y (2013) BrainNet Viewer: A Network Visualization Tool for Human Brain Connectomics. PLoS One.

Xiong Y, Mahmood A, Chopp M (2013) Animal models of traumatic brain injury. Nat Rev Neurosci.

Yeh FC, Verstynen TD, Wang Y, et al. (2013) Deterministic diffusion fiber tracking improved by quantitative anisotropy. PLoS One 8:1-16.

Yuan W, Wade SL, Babcock L (2015) Structural connectivity abnormality in children with acute mild traumatic brain injury using graph theoretical analysis. Hum Brain Mapp 36:779-792.

Zalesky A, Fornito A, Bullmore ET (2010) Network-based statistic: Identifying differences in brain networks. Neuroimage 53:1197-1207 Available at: http://dx.doi.org/10.1016/j.neuroimage.2010.06.041. 